\title{
Discontinuous Feedback Stabilization of the Angular Velocity of a Rigid Body with Two Control Torques
}

\author{
Mahmut Reyhanoglu \\ Department of Applied Mathematics \\ University of Twente \\ The Netherlands \\ m.reyhanoglu@math.utwente.nl
}

\begin{abstract}
There has been much interest over the past decade in the problem of asymptotic stabilization of the angular velocity of a rigid body with only two torque inputs. The smooth feedback laws proposed in the literature provide asymptotic stability with nonexponential convergence rates. This paper proposes discontinuous feedback laws to achieve asymptotic stability with exponential convergence rates.
\end{abstract}

\section{Introduction}

The angular velocity control of a rigid body with only one or two controls has been studied extensively in the literature. In particular, many strategies have been proposed for the stabilization and asymptotic stabilization problems. The asymptotic stabilization problem for the case of a single control aligned with an axis having components along all three principal axes was studied in [4], [7], [9]. In [5], it was shown by finding a Lyapunov function that the null solution of the angular velocity equations is asymptotically stabilizable by two control torques aligned with two principal axes if the uncontrolled axis is not an axis of symmetry. The same result was demonstrated in [3] using center manifold theory.

In this paper, it is first shown that the angular velocity equations of a rigid body with two control torques cannot be exponentially stabilized using any $C^{1}$ feedback law, and hence, the rates of convergence provided by the smooth feedback laws proposed in the literature (see e.g. [3], [5], and references therein) are necessarily nonexponential. Discontinuous feedback laws are then constructed to achieve asymptotic stabilization with exponential convergence rates. The methodology followed in the construction of the discontinuous feedback laws is based on first transforming the nonlinear control system describing the evolution of the angular velocity into a discontinuous system which is exponentially stabilizable via smooth feedback laws, and then designing exponentially stabilizing feedback laws for the transformed system. Finally, transforming back into the o0-7803-3590-2/96\$5.00 ๔ 1996 IEEE riginal coordinates yields asymptotically stabilizing discontinuous feedback laws with exponential convergence rates for the original system. This construction procedure is related to the approaches proposed in [2], [8] for the stabilization of nonholonomic systems.

\section{Problem Formulation}

Consider a rigid body which is controlled by means of two torque inputs applied about two principal axes. Let $\omega_{1}, \omega_{2}, \omega_{3}$ be the angular velocity components with respect to the principal axes, and denote by $j_{1}, j_{2}, j_{3}$ the respective principal moments of inertia. For simplicity, assume that the two torque inputs are about the first two principal axes. Then the Euler's equations of motion of the rigid body are given by

$$
\begin{aligned}
& j_{1} \dot{\omega}_{1}=\left(j_{2}-j_{3}\right) \omega_{2} \omega_{3}+\tau_{1}, \\
& j_{2} \dot{\omega}_{2}=\left(j_{3}-j_{1}\right) \omega_{3} \omega_{1}+\tau_{2}, \\
& j_{3} \dot{\omega}_{3}=\left(j_{1}-j_{2}\right) \omega_{1} \omega_{2},
\end{aligned}
$$

where $\tau_{1}, \tau_{2}$ are the torque inputs.

Applying the feedback transformation

$u_{1}=\frac{j_{2}-j_{3}}{j_{1}} \omega_{2} \omega_{3}+\frac{\tau_{1}}{j_{1}}, u_{2}=\frac{j_{3}-j_{1}}{j_{2}} \omega_{3} \omega_{1}+\frac{\tau_{2}}{j_{2}}$,

yields

$\dot{\omega}_{1}=u_{1}, \dot{\omega}_{2}=u_{2}, \dot{\omega}_{3}=a \omega_{1} \omega_{2}$,

where $a=\left(j_{1}-j_{2}\right) / j_{3}$.

Clearly, (4) defines a nonlinear control system with state $\omega=\left(\omega_{1}, \omega_{2}, \omega_{3}\right) \in R^{3}$ and control $u=\left(u_{1}, u_{2}\right) \in$ $R^{2}$.

Consider the problem of designing state feedback laws $u=u(\omega)$ which asymptotically stabilizes the system (4) to the origin. This problem is of important practical interest since the designed state feedback laws can be used to asymptotically stabilize the angular velocity of a rigid body, for instance a rigid spacecraft, in an actuator failure mode. Clearly, if the uncontrolled principal axis is an axis of symmetry of the rigid body, i.e. if $j_{1}=j_{2}$, then $\omega_{3}(t)=\omega_{3}(0), \forall t \geq 0$. This means 
that $\omega_{3}$ is a constant of the motion. In this case, the system is not even accessible. In particular, if $\omega_{3}(0) \neq 0$, then $\omega_{3}$ cannot be transferred to zero using any control function. In this paper, it is assumed that the uncontrolled principal axis of the rigid body is not an axis of symmetry, i.e. $j_{1} \neq j_{2}$.

The following result is a consequence of the fact that a smooth nonlinear control system is exponentially stabilizable using $C^{1}$ feedback if and only if its linearization is stabilizable [10].

Proposition 1: The angular velocity equations of a rigid body with only two control torques aligned with two principal axes cannot be exponentially stabilized using $C^{1}$ feedback law.

Proof: It is easily verified that the linearization of the system (4) about the origin has an uncontrollable eigenvalue at the origin. Hence, the linearized system is not stabilizable. Thus, the system (4) violates the necessary condition for the existence of exponentially stabilizing $C^{1}$ feedback [10]. It follows that the angular velocity of a rigid body with only two control torques aligned with two principal axes cannot be exponentially stabilized using $C^{1}$ feedback law.

The above result demonstrates that the rates of convergence provided by smooth feedback laws proposed in the literature are necessarily nonexponential. Furthermore, one can show that smooth feedback laws do not transfer the velocity of the rigid body to the origin in a reasonable amount of time. Thus, feedback laws which provide faster convergence rates are desirable. Next section demonstrates that exponential convergence rates are achievable via discontinuous feedback laws.

\section{Discontinuous Feedback Laws}

The idea employed in this section is based on first transforming the system (4) into a discontinuous one by applying a discontinuous coordinate transformation, e.g. by applying a $\sigma$-process [1].

Possible $\sigma$-processes for the system (4) are given by the passage from the coordinates $\left(\omega_{1}, \omega_{2}, \omega_{3}\right)$ to the coordinates $\left(\omega_{1}, \omega_{2}, \omega_{3} / \omega_{1}\right)$, where $\omega_{1} \neq 0$, and to the coordinates $\left(\omega_{1}, \omega_{2}, \omega_{3} / \omega_{2}\right)$, where $\omega_{2} \neq 0$.

Consider the system (4). Restricting consideration to $\omega_{1}^{2}+\omega_{2}^{2} \neq 0$, stabilizing feedback control laws can be constructed as follows:

For the case when $\omega_{1} \neq 0$, applying the $\sigma$-process $x_{1}=\omega_{1}, x_{2}=\omega_{2}, x_{3}=\frac{\omega_{3}}{\omega_{1}}$,

we obtain

$\dot{x}_{1}=u_{1}, \dot{x}_{2}=u_{2}, \dot{x}_{3}=a x_{2}-\frac{x_{3}}{x_{1}} u_{1}$.
Clearly, the feedback control law

$\left(u_{1}(x), u_{2}(x)\right)=\left(-k_{1} x_{1},-k_{2} x_{2}-k_{3} x_{3}\right)$,

where $k_{i}, i=1,2,3$ are the gains, yields the closed-loop system

$\dot{x}_{1}=-k_{1} x_{1}$,

$\dot{x}_{2}=-k_{2} x_{2}-k_{3} x_{3}$,

$\dot{x}_{3}=a x_{2}+k_{1} x_{3}$

Similarly, for the case when $\omega_{2} \neq 0$, applying the $\sigma$-process

$x_{1}=\omega_{1}, x_{2}=\omega_{2}, x_{3}=\frac{\omega_{3}}{\omega_{2}}$,

we obtain

$\dot{x}_{1}=u_{1}, \dot{x}_{2}=u_{2}, \dot{x}_{3}=a x_{1}-\frac{x_{3}}{x_{2}} u_{2}$.

Clearly, the feedback control law

$\left(u_{1}(x), u_{2}(x)\right)=\left(-k_{2} x_{1}-k_{3} x_{3},-k_{1} x_{2}\right)$,

where $k_{i}, i=1,2,3$ are the gains, yields the closed-loop system

$\dot{x}_{1}=-k_{2} x_{1}-k_{3} x_{3}$,

$\dot{x}_{2}=-k_{1} x_{2}$

$\dot{x}_{3}=a x_{1}+k_{1} x_{3}$.

Note that the two closed-loop systems corresponding to the first and second $\sigma$-processes are exponentially stable when $k_{1}>0$ and $k_{2}$ and $k_{3}$ are chosen such that the matrix

$A=\left(\begin{array}{cc}-k_{2} & -k_{3} \\ a & k_{1}\end{array}\right)$

is a Hurwitz matrix. It is clear that the spectrum of the matrix $A$ can be assigned arbitrarily through $k_{2}$ and $k_{3}$.

Note also that in the original coordinates the two closed-loop systems corresponding to the first and second $\sigma$-processes are given as follows:

$\dot{\omega}_{1}=-k_{1} \omega_{1}$

$\dot{\omega}_{2}=-k_{2} \omega_{2}-k_{3} \frac{\omega_{3}}{\omega_{1}}$,

$\dot{\omega}_{3}=a \omega_{1} \omega_{2}$

and

$\dot{\omega}_{1}=-k_{2} \omega_{1}-k_{3} \frac{\omega_{3}}{\omega_{2}}$,

$\dot{\omega}_{2}=-k_{1} \omega_{2}$

$\dot{\omega}_{3}=a \omega_{1} \omega_{2}$. 
The following result can now be stated.

Proposition 2: Consider the system (4) with the feedback law

$u(\omega)= \begin{cases}\left(-k_{1} \omega_{1},-k_{2} \omega_{2}-k_{3} \frac{\omega_{3}}{\omega_{1}}\right) & \text { if } \omega_{1} \neq 0, \\ \left(-k_{2} \omega_{1}-k_{3} \frac{\omega_{3}}{\omega_{2}},-k_{1} \omega_{2}\right) & \text { otherwise, }\end{cases}$

with $k_{1}>0$ and $k_{2}$ and $k_{3}$ are such that the matrix $A$ given by (15) is a Hurwitz matrix. Let $\left(\omega_{10}, \omega_{20}, \omega_{30}\right)$ denote an initial condition with $\omega_{10}^{2}+\omega_{20}^{2} \neq 0$. Then the following hold.

(i) The trajectory $\left(\omega_{1}(t), \omega_{2}(t), \omega_{3}(t)\right)$ is bounded for all $t \geq 0$ and converges exponentially to zero.

(ii) The control $\left(u_{1}(t), u_{2}(t)\right)$ is bounded for all $t \geq 0$ and converges exponentially to zero.

Proof: Consider the system (4) with the feedback law (22) and let $\left(\omega_{10}, \omega_{20}, \omega_{30}\right)$ denote an initial condition with $\omega_{10}^{2}+\omega_{20}^{2} \neq 0$.

It is clear that if $\omega_{1} \neq 0$, then the closed-loop system is given by (16)-(18), otherwise by (19)-(21).

(i) First assume that $\omega_{10} \neq 0$. Then from equations (16)-(18), we have $\omega_{1}(t)=\omega_{10} e^{-k_{1} t}$. Clearly, $\omega_{1}(t) \neq$ $0, \forall t \in[0, \infty)$. Hence, in this case, the closed-loop system is given by (16)-(18) (or equivalently by (7)-(9) in the transformed coordinates) for all $t \geq 0$. Since the matrix $A$ is Hurwitz, it follows that $\left(x_{2}(t), x_{3}(t)\right)$, and hence $\left(\omega_{2}(t), \omega_{3}(t) / \omega_{1}(t)\right)$, converges exponentially to zero. It follows that, if $\omega_{10} \neq 0$, then the trajectory $\left(\omega_{1}(t), \omega_{2}(t), \omega_{3}(t)\right)$ is bounded for all $t \geq 0$ and converges exponentially to zero.

On the other hand, if $\omega_{10}=0$, then from equations (19)-(21), we have $\omega_{2}(t)=\omega_{20} e^{-k_{1} t}$. Clearly, $\omega_{2}(t) \neq 0, \forall t \in[0, \infty)$. Hence, in this case, the closedloop system is given by (19)-(21) (or equivalently by (12)-(14) in the transformed coordinates) for all $t \geq 0$. Again since the matrix $A$ is Hurwitz, it follows that $\left(x_{2}(t), x_{3}(t)\right)$, and hence $\left(\omega_{1}(t), \omega_{3}(t) / \omega_{2}(t)\right)$, converges exponentially to zero. Thus, if $\omega_{10}=0$, then the trajectory $\left(\omega_{1}(t), \omega_{2}(t), \omega_{3}(t)\right)$ is bounded for all $t \geq 0$ and converges exponentially to zero.

(ii) From the above discussions, each term in the control (22) consists of bounded terms which converge exponentially to zero. Hence, the result follows.

Remark 1: The above result demonstrates that for initial conditions satisfying $\omega_{10}^{2}+\omega_{20}^{2} \neq 0$, the feedback control law (22) is well-defined for all $t \geq 0$. Moreover, it drives the system (4) to the origin, while avoiding the set

$N=\left\{\omega \in \mathbf{R}^{3} \mid \omega_{1}^{2}+\omega_{2}^{2}=0, \omega_{3} \neq 0\right\}$.

Clearly, one can use a finite time feedback control law to move the system away from $N$. For example,

$u_{i}=-\left|\omega_{i}-\epsilon_{i}\right|^{\alpha} \operatorname{sign}\left(\omega_{i}-\epsilon_{i}\right), i=1,2$, where $\alpha \in[0,1), \epsilon_{1}$ and $\epsilon_{2}, \epsilon_{1}^{2}+\epsilon_{2}^{2} \neq 0$, are constants, can be used to move the system to $\omega_{1}=\epsilon_{1}, \omega_{2}=\epsilon_{2}$ in finite time [6].

Remark 2: The discontinuous control law (22) is not an exponential stabilizer for the system (4). It only guarantees exponential convergence of the closed-loop state and control trajectories for initial conditions satisfying $\omega_{10}^{2}+\omega_{20}^{2} \neq 0$.

\section{Conclusions}

The problem of asymptotic stabilization of the angular velocity of a rigid body with only two control torques aligned with two principal axes has been considered. Discontinuous feedback laws have been proposed to achieve exponential convergence rates. Boundedness and exponential convergence of the closed-loop state and control trajectories have been demonstrated.

Acknowledgement- The author wishes to acknowledge the support provided by the Dutch Institute of Systems and Control that enabled his visit to the University of Twente during the academic year 1995-1996.

\section{References}

[1] V.I. Arnold, Gcometrical Methods in the Theory of Ordinary Differential Equations, Springer-Verlag, New York Inc., 1983.

[2] A. Astolfi, "Exponential Stabilization of Nonholonomic Systems via Discontinuous Control," Nonlinear Control System Design Symposium (NOLCOS), IFAC, Tahoe City, 1995, pp.741-746.

[3] D. Aeyels, "Stabilization of a Class of Nonlinear Systems by a Smooth Feedback Control," Systems and Control Letters, 5(5), 1985, pp.289-294.

[4] D. Aeyels and M. Szafranski, "Comments on the Stabilizability of the Angular Velocity of a Rigid Body," Systems and Control Letters, 10(1), 1988, pp.35-39.

[5] R.W. Brockett, "Asymptotic Stability and Feedback Stabilization,"in Differential Geometric Control Theory, R.W. Brockett, R.S. Millman and H.J. Sussmann, eds., Birkhauser, Boston, 1983, pp.181-191.

[6] V.T. Haimo, "Finite Time Controllers," SIAM J. Control and Optimization, 24(4), 1986, pp.760-770.

[7] R. Outbib and G. Sallet, "Stabilizability of the Angular Velocity of a Rigid Body Revisited," Systems and Control Letters, 18(2), 1992, pp.93-98.

[8] M. Reyhanoglu, "On the Stabilization of a Class of Nonholonomic Systems Using Invariant Manifold Technique," Proceedings of IEEE Conference on Decision and Control, 1995, New Orleans, LA, pp.2125-2126.

[9] E.D. Sontag and H.J. Sussmann, "Further Comments on the Stabilizability of the Angular Velocity of a Rigid Body Revisited," Systems and Control Letters, 12(3), 1988, pp.213-217.

[10] J. Zabczyk, "Some Comments on Stabilizability," Applied Mathematics and Optimization, 19, 1989, pp.1-9. 